Memorias del VII Encuentro Nacional de Experiencias en la Enseñanza de la

Biología y la Educación Ambiental y II Congreso Nacional de Investigación en la Enseñanza de la Biología

\title{
LA ENSEÑANZA DE LA BIOLOGÍA MOLECULAR A TRAVÉS DE PRÁCTICAS DE LABORATORIO EN LA LICENCIATURA EN BIOLOGÍA DE LA UNIVERSIDAD PEDAGÓGICA NACIONAL -CENTRO VALLE DE TENZA. (SUTATENZA - BOYACÁ).
}

\section{TEACHING OF MOLECULAR BIOLOGY WITH LABORATORY PRACTICE IN THE DEGREE OF BIOLOGY UNIVERSIDAD PEDAGOGICA NACIONAL - CENTRO VALLE DE TENZA.(SUTATENZA - BOYACÁ).}

\section{Leidy Tatiana García Navarrete ${ }^{1}$}

\section{Resumen}

Este artículo presenta el trabajo realizado en práctica pedagógica en modalidad integral, durante el primer semestre del 2013, con 12 estudiantes del componente Biología Molecular de la Licenciatura en Biología de la Universidad Pedagógica Nacional (UPN), extensión Centro Valle de Tenza (CVT) ubicado en el municipio de Sutatenza (Boyacá).

El trabajo consto de cuatro fases orientadas por la Línea de Investigación Enseñanza de la Biotecnología en Colombia de la UPN, las cuales tuvieron como propósito contribuir a la enseñanza de la Biología Molecular a través de prácticas de laboratorio (virtuales, convencionales y artesanales), asumiéndose como estrategia didáctica.

En la primera fase se encontró; que para los estudiantes las prácticas de laboratorio les permiten un acercamiento y fortalecimiento de los elementos teóricos, llevándose la teoría a la práctica. Sin embargo, fueron casi nulas en su formación previa a la universitaria. En la segunda fase se realizaron actividades que buscaron el fortalecimiento del Laboratorio de Biología del CVT, destacándose la realización del Manual de Bioseguridad del Laboratorio de Biología y el apoyo al trabajo de la Ludoteca UPN-CVT.

La tercera y cuarta fase consistieron en la implementación y análisis de los tres tipos de prácticas de laboratorio (virtuales, convencionales y artesanales), que se configuraron como una tripleta funcional, donde sus ventajas se complementan y hacen significativo el proceso de aprendizaje de temáticas en torno a la Biología Molecular en contextos rurales.

\footnotetext{
${ }^{1}$ Estudiante IX semestre de la Licenciatura en Biología Universidad Pedagógica Nacional - sede Bogotá Itgarcian@gmail.com, dbi.Igarcia@pedagogica.edu.co
} 
Bio-grafía Escritos sobre la Biología y su Enseñanza.

Edición Extra-Ordinaria. ISSN 2027-1034 P.p 291 - 299

Memorias del VII Encuentro Nacional de Experiencias en la Enseñanza de la

Biología y la Educación Ambiental y II Congreso Nacional de Investigación en la Enseñanza de la Biología

Palabras claves: Prácticas de laboratorio, enseñanza de la Biología Molecular, estrategia didáctica.

\section{Abstract}

This article presents the work done in teaching practice based on integrated mode, during the first period of 2013, with 12 students of the Molecular component in Biology degree from Universidad Pedagógica Nacional (UPN), extension Centro Valle de Tenza (CVT) located in the township of Sutatenza (Boyacá).

This work had four phases, they were oriented by Teaching Research Line of Biotechnology in Colombia of the UPN, which had as a purpose to contribute to the teaching of molecular biology through laboratory practice (virtual, conventional and artisanal), and those were assumed as a teaching strategy.

In the first phase has found that the laboratory practices permit an approach and a strengthening of the theoretical elements to the students, taking the theory into practice. However, those were almost nil in their pre-university education. The second phase had different activities that sought to strengthen of the Biology Laboratory of CVT, emphasizing in the realization of the Laboratory Biosafety Manual Biology and supporting the work of the ludoteca UPN-CVT.

The third and fourth phase consisted of the implementation and analysis of the three types of laboratory practice (virtual, conventional and artisanal), which were configured as a functional triplet, where their advantages supplemented and do significant the learning process of thematics of Molecular Biology on rural contexts.

Keywords: Laboratory practice, Molecular Biology teaching and teaching strategy.

\section{Introducción}

Diferentes trabajos se han desarrollado en torno a la importancia, las limitaciones y las ventajas de incluir en la enseñanza de las ciencias los trabajos prácticos (TP), los cuales de acuerdo a Correa (2012) son una línea de investigación del campo de conocimiento que se ocupa de la Enseñanza de la Biología, donde autores como Barrerá \& Valdés (1996), Puentes (2008) y Correa (2012) agrupan las salidas de campo, las prácticas de laboratorio, las actividades de lápiz y papel, entre otras.

Para el presente trabajo se centra la atención en las prácticas de laboratorio (PL), las cuales se contextualizan dentro del campo de la Biología Molecular, puesto que "ha construido una nueva concepción de lo vivo, que se basa en lo "invisible", donde las interpretaciones teóricas se apoyan en métodos experimentales 
Bio-grafía Escritos sobre la Biologia y su Enseñanza.

Edición Extra-Ordinaria. ISSN 2027-1034 P.p 291-299

Memorias del VII Encuentro Nacional de Experiencias en la Enseñanza de la

Biología y la Educación Ambiental y 11 Congreso Nacional de Investigación en la

Enseñanza de la Biología

deductivos e inductivos, que permiten la comprensión de los complejos fenómenos vivos ocurrentes a nivel intracelular" (Castro, 2011).

Por lo tanto, para conocer este mundo invisible, de acuerdo con Correa (2012) el empleo de prácticas de laboratorio contribuirán al estudio de fenómenos biológicos a nivel microscópico, a la enseñanza de conceptos difíciles de aprender, y la posibilidad de la enseñanza desde un punto de vista multidisciplinar, argumentos compartidos con Córdoba (2012) y enfatizados al relacionarlos con las implicaciones de la enseñanza de la Biología Molecular, al presentar un alto nivel de especificación y complejidad en los conceptos.

De acuerdo con los argumentos anteriormente expuestos, se plantea la siguiente pregunta problema: ¿Por qué a través de las prácticas de laboratorio (Virtual, artesanal y convencional) se contribuye en la enseñanza de la Biología Molecular en los estudiantes de VII semestre de la licenciatura en Biología Universidad Pedagógica Nacional -Centro Valle de Tenza?

\section{Aspecto Metodológico}

La investigación se orientó bajo un enfoque cualitativo, asumido desde Bonilla \& Rodriguez (1997)al centrarse en la exploración de manera sistemática de los conocimientos y valores que comparten los individuos en un determinado contexto espacial y temporal. El método se asumió desde la inducción al permitir "pasar de los hechos particulares a los principios generales. Partiendo de la observación de múltiples hechos o fenómenos para luego clasificarlos y llegar a establecer las relaciones o puntos de conexión entre ellos" (Hurtado \& Toro, 2007).

Con la finalidad de registrar y analizar el proceso de enseñanza de la Biología Molecular en un contexto rural emplearon entrevistas semi-estructuras, observación participante, cuestionarios, discusiones, reflexiones, prácticas de laboratorio y salida de campo. Las cuales se desarrollaron en 4 fases (contextualización, aporte a la consolidación del laboratorio de Biología, ejecución de prácticas de laboratorio y análisis de resultados), que son permeadas por la reflexión en torno a la enseñanza de la Biología, representada en la parte central de la figura 1. 
Bio-grafía Escritos sobre la Biología y su Enseñanza.

Edición Extra-Ordinaria. ISSN 2027-1034 P.p 291-299

Memorias del VII Encuentro Nacional de Experiencias en la Enseñanza de la Biología y la Educación Ambiental y II Congreso Nacional de Investigación en la Enseñanza de la Biología

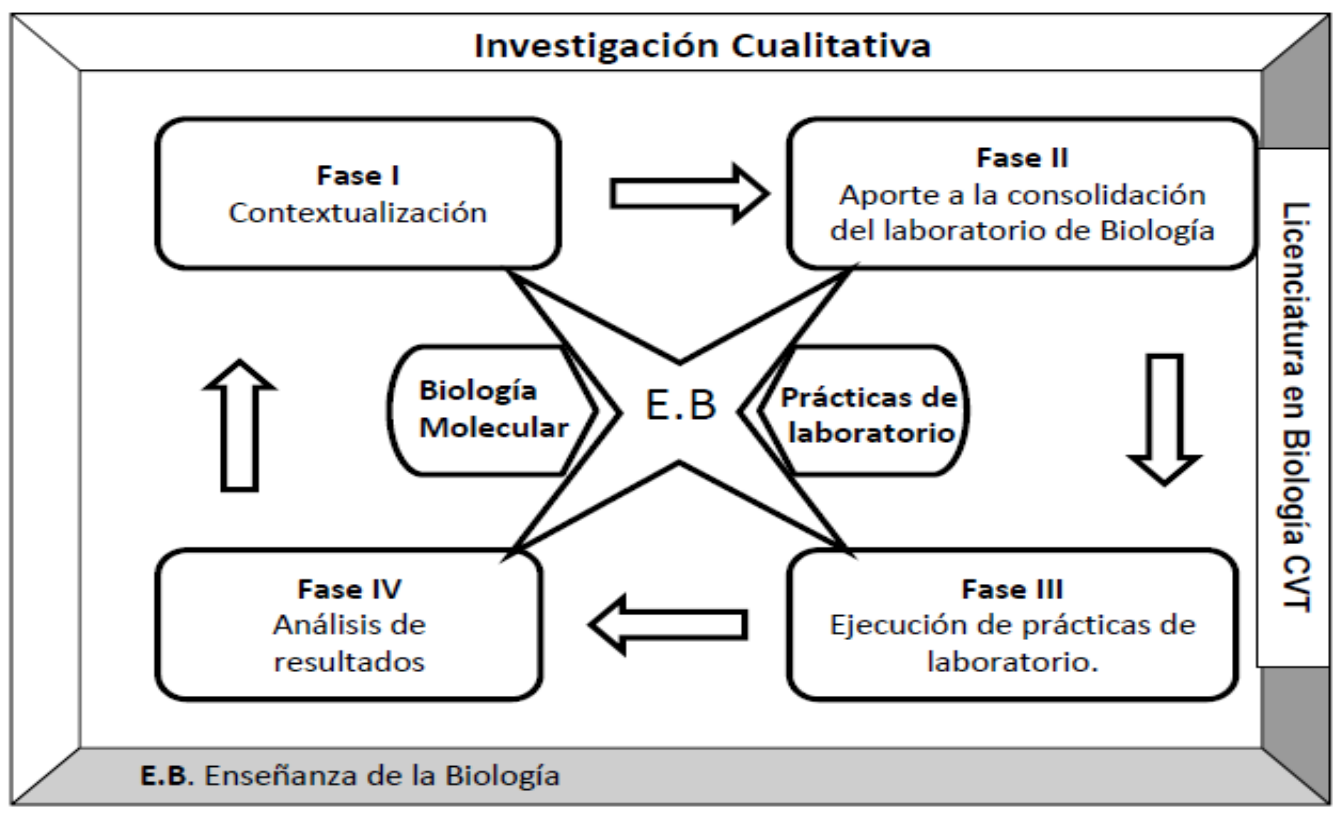

Figura 1. Fases de la investigación.

\section{Resultados Y Discusión De Los Resultados.}

Durante la primera fase, se encontró una marcada coincidencia entre los argumentos de Barrerá y Valdés (1996) y los expuestos por los estudiantes en la indagación de ideas previas, en torno a las prácticas de laboratorio. Puesto que asumen este tipo de trabajo practico, como el momento de acercamiento y fortalecimiento de los elementos teóricos mediante la práctica, limitándose como lo plantean Barrerá y Valdés (1996) al aprendizaje de técnicas experimentales, comprobación y refuerzo de teorías.

Las prácticas de laboratorio que predominaron en el ciclo de formación previo al universitario estuvieron centradas en el componente de Biología, seguido del de física y química. Donde la práctica más realizada fue la observación de células vegetales (cebolla). Este elemento permite reflexionar de acuerdo a Puentes (2008) y Valbuena (2007) en torno a la manera en que se han abordado los trabajos prácticos en la escuela, y el papel del maestro al plantear, diseñar y orientar los trabajos prácticos de los estudiantes, resaltando la importancia de incluir y fortalecer en la formación de los licenciados la realización de diversas prácticas de laboratorio, que les permita diseñar material didáctico para las necesidades del contexto rural.

En la fase II denominada, apoyo a la consolidación del laboratorio de Biología del CVT, se realizaron trabajos de apropiación con los estudiantes del componente de 
Memorias del VII Encuentro Nacional de Experiencias en la Enseñanza de la Biología y la Educación Ambiental y II Congreso Nacional de Investigación en la

\section{Enseñanza de la Biología}

Biología Molecular y los niños de la ludoteca de la UPN-CVT, en torno a los equipos del laboratorio, y las normas de Bioseguridad. Puesto que las prácticas de laboratorios se deben partir con "la familiarización del equipo, normas y protocolos de seguridad” (Córdoba, 2012).

En cuanto a los estudiantes de séptimo semestre, estos reflexionaron en torno a la importancia de las normas de bioseguridad al ser llevadas a la escuela y asumieron su rol como docentes responsables y orientadores de este tipo de actividades.

E5-E9:"por otra parte la implementación de las normas de bioseguridad en las prácticas de laboratorio con estudiantes exige una mayor rigurosidad en su cumplimiento ya que los niños - jóvenes son más inquietos y suelen estar cogiendo y moviendo los materiales e implementos del mismo laboratorio ocasionando un posible accidente así mismo o al grupo $(\ldots)$.
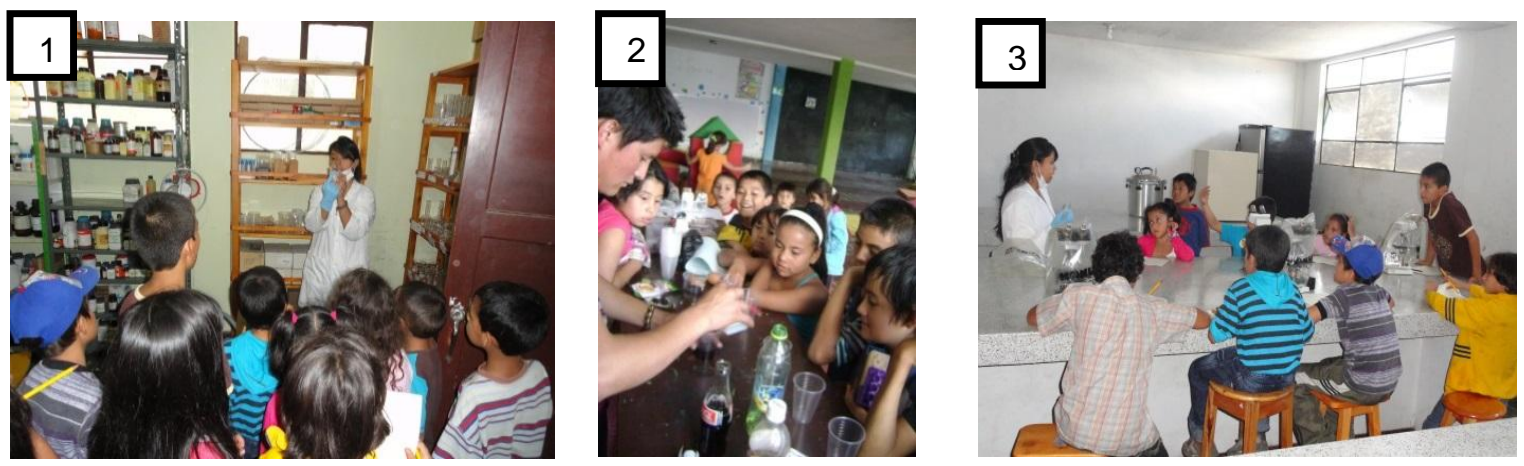

Imágenes 1 - 3. Tomadas por: Faldiño, C. (2013)

Mientras que él apoyó al espacio de ciencias de la ludoteca UPN-CVT consistió en actividades sobre combustión de cloruros, siembra de bacterias de la boca, el reconocimiento de los equipos e instrumentos del laboratorio, normas de bioseguridad y observación del cultivo de bacterias bucales e insectos. (Ver imágenes 1-3). Actividades que buscaron de acuerdo con Alfonso \& Alfonso (2012) potencializaren los niños el desarrollo de habilidades, como la observación y la descripción, además de motivarlos a despertar el gusto por la ciencias.

El último aporte en esta fase consistió en la elaboración del Manual de Bioseguridad del Laboratorio de Biología UPN-CVT, que pretendió a través del tercer principio de enseñanza de Wilson (2006), y la inclusión de elementos históricos desde Ramírez \& Cardona (2010)presentar de manera sencilla algunos elementos en torno al desarrollo de prácticas de laboratorio seguras, brindándoles a los estudiantes de la licenciatura en Biología y demás usuarios del laboratorio 
Memorias del VII Encuentro Nacional de Experiencias en la Enseñanza de la

Biología y la Educación Ambiental y II Congreso Nacional de Investigación en la

$$
\text { Enseñanza de la Biología }
$$

información clave acerca de bioseguridad, además del reconocimiento y manejo de los equipos del laboratorio.

A continuación se expone el desarrollo de los tres tipos de prácticas de laboratorio, virtuales, convencionales y artesanales, centrales en la tercera fase.

Con la implementación de los laboratorios virtuales, los estudiantes las asumieron como un acercamiento a los conocimientos disciplinares y a las herramientas tecnológicas. Reconocen el aporte a la comprensión general de las técnicas estudiadas (extracción de ADN y electroforesis) y su potencial uso en su futuro profesional a nivel de básica y media, haciendo énfasis en las nomas de bioseguridad casi nulas en los simuladores. Por consiguiente, los simuladores de laboratorio se convierten en un recurso importante en un contexto rural, al solventar de acuerdo con Córdoba (2012) necesidades didácticas, técnicas y económicas de las instituciones educativas.

En cuanto a las falencias, los maestros en formación destacan que los simuladores se encuentran en idioma inglés, sin embargo a través de los estos se puede potencializar el desarrollo de habilidades comunicativas en lengua extranjera. Otras falencias giran en torno a la limitada experiencia y modificación de variables que presentan los simuladores, además de su superficialidad para el nivel universitario, elementos compartidos con Córdoba (2012).Bajo esta mirada se reitera la invitación de Valbuena (2007) a que los maestros elaboren material didáctico contextualizado y se involucren en la valoración, modificación e implementación de los materiales tradicionalmente empleados y reflexione estos elementos al proponer nuevos materiales.

Durante la salida de campo a Bogotá (ver imagen 4) al Instituto SINCHI, al grupo de investigación de Biología Molecular de la Universidad Francisco José de Caldas y la línea de investigación Enseñanza de la Biotecnología en Colombia de la UPN, los estudiantes realizaron diferentes prácticas de laboratorio convencionales, que les produjeron diversas emociones, las cuales de acuerdo con Wilson (2006) generaran aprendizajes, permitiendo que el conocimiento no los abandone jamás.E5: "el poder tener mi ADN en mis manos, no tiene descripción y la experiencia que realizamos este día, fue para no olvidar y querer replicar".

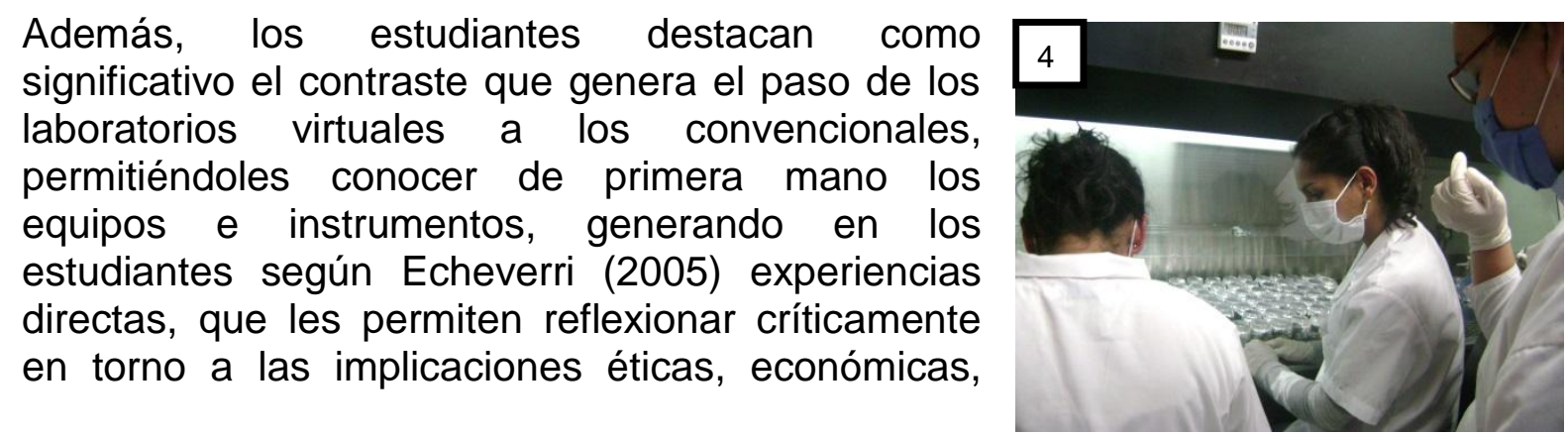


Memorias del VII Encuentro Nacional de Experiencias en la Enseñanza de la Biología y la Educación Ambiental y II Congreso Nacional de Investigación en la

\section{Enseñanza de la Biología}

sociales y culturales de la enseñanza de la biotecnología en contextos rurales, planteándose de acuerdo a Roa (2010) la necesidad de la constante formación de los maestros en temáticas biotecnológicas y su didáctica.

Frente a los laboratorios artesanales y los montajes de equipos artesanales (centrifuga, cámara de electroforesis, trans-iluminador) los estudiantes destacan su importancia para el fortalecimiento de los conocimientos conceptuales y procedimentales. Convirtiéndose en una alternativa novedosa, debido a que se realizar con "elementos prácticos que se pueden encontrar al alcance de la mano".

De esta manera el montaje artesanal facilito el acercamiento a las dinámicas submicroscopicas, puesto que "la biología molecular ha construido una nueva concepción de lo vivo, que se basa en lo "invisible"(Castro, 2011), por lo tanto estos montajes se configuran como una estrategia didáctica que contribuye en la formación del licenciado en biología al producir herramientas propias para su aprendizaje.

Por último, se debe considerar que los tres tipos de prácticas de laboratorio aportan a la formación de los futuros licenciados en Biología al denominarse por el E6 como una tripleta funcional que desde sus ventajas complementan y hacen significativo el proceso de aprendizaje; Respondiéndose de esta manera a las características del contexto e invitando al maestro en formación a la implementación de prácticas de laboratorio durante su ejercicio docente.

\section{Conclusiones}

La implementación conjunta de prácticas de laboratorio virtuales, convencionales y artesanales, se asume como una estrategia didáctica positiva que potencializó el proceso de aprendizaje de los futuros maestros, al permitirles de acuerdo a Correa (2012) y Córdoba (2012) relacionarse con conceptos abstractos y técnicas específicas desde una perspectiva multidisciplinar. Donde las prácticas de laboratorios virtuales y artesanales se configuran como una alternativa de trabajo para la implementación en contextos rurales.

En cuanto a la transformación del laboratorio de Biología de la UPN-CVT, este se resignificó como un espacio dinámico y reflexible que contribuye al aprendizaje de los estudiantes de diferentes niveles educativos, proyectando una perspectiva intercultural para la enseñanza de las ciencias, mediada por relaciones de inclusión y correspondencia entre los diversas estructuras ideologías propias del contexto rural.

Finalmente, se debe destacar el gran valor e importancia de la práctica pedagógica en modalidad integral apoyando la docencia universitaria, la cual le permite al maestro practicante reflexionar en torno a los diferentes conocimientos 
Bio-grafía Escritos sobre la Biología y su Enseñanza.

Edición Extra-Ordinaria. ISSN 2027-1034 P.p291-299

Memorias del VII Encuentro Nacional de Experiencias en la Enseñanza de la Biología y la Educación Ambiental y II Congreso Nacional de Investigación en la

\section{Enseñanza de la Biología}

profesionales del profesor, asumidos desde Valbuena (2007), (conocimiento disciplinar, pedagógico, contextual y didáctico del contenido disciplinar), al llevarlo a cuestionarse constantemente sobre su ser y quehacer como maestro, en un país con un carácter pluriétnico y multicultural, donde el abordaje de temáticas biotecnológicas se convierte en un reto profesional.

\section{Bibliografía}

Alfonso, M., \& Alfonso, V. (2012). Sutatenza involucrada a la familia, con niños y niñas menores de 5 años en la educación inicial. Sutatenza (Boyacá): Universidad Pedagógica Nacional.

Barrerá, O., \& Valdés, P. (1996). El trabajo practico en la enseñanza de las ciencias: una revisión. Enseñanza de las Ciencias., 365-379.

Bonilla, E., \& Rodriguez, P. (1997). Más allá del dilema de los métodos: La investigación en ciencias sociales. Bogotá: Norma.

Castro, J. (2011). El modelo del operón Lac1 50 años depués. ¿Qué implicaciones tiene en la enseñanza de la biología hoy? Bio-grafía:Escritos sobre la Biología y su Enseñanza., 100-110.

Córdoba, L. (2012). Valoración de la usabilidad técnica y didáctica de los simuladores de laboratorio virtual para la Biología Molecular DBI-UPN. Bogotá: Universidad Pedagógica Nacional.

Correa, M. (2012). Estado del arte sobre los trabajos prácticos en la enseñanza de la biología (2004-2008): un aporte a la formación docente. Bogotá: Universidad Pedagógica Nacional.

Echeverri, X. (2005). Reflexión sobre la facilitación del aprendizaje experiencial. Internacional del Magisterio, Educación y Pedagogía.

Hurtado, I., \& Toro, J. (2007). Paradigmas y métodos de investigacién en tiempos de cambio. Modelos de conocimiento que rigen los procesos de investigación y los métodos científicos expuestos desde la perspectiva de las Ciencias Sociales. Venezuela: Libros El Nacional. 
Bio-grafía Escritos sobre la Biología y su Enseñanza.

Edición Extra-Ordinaria. ISSN 2027-1034 P.p291-299

Memorias del VII Encuentro Nacional de Experiencias en la Enseñanza de la

Biología y la Educación Ambiental y II Congreso Nacional de Investigación en la Enseñanza de la Biología

Puentes, M. (2008). Propuesta de un sistema de categorías para el estudio del trabajo práctico en la enseñanza de la Biología. Bogotá: Universidad Pedagógica Nacional.

Ramírez , N., \& Cardona, V. (2010). Importancia de incluir la enseñanza de la Historia, la filosofía y la epistemología en las clases de ciencias. Asociación Colombiana para la investigación en Ciencias y tecnología EDUCyT. Memoria II Congreso Nacional de investigación en Ciencia y Tecnología.

Roa, R. (2010). Referentes de la Biotecnología para la enseñanza de las Ciencias Naturales. Bio-grafía: Escritos sobre Biología y su Enseñanza., 511-521.

Valbuena, E. (2007). El Conocimiento Didáctico del Contenido Biológico. Estudio de las concepciones disciplinares y didácticas de futuros docentes de la Universidad Pedagógica Nacional (Colombia). Madrid., España: Universidad Complutense de Madrid.

Wilson, E. (2006). La creación. Salvemos la vida en la tierra. Buenos Aires : Katz Editores. 